\title{
BRINGING PATIENT NAVIGATION TO PRIMARY HEALTH CARE IN RIO DE JANEIRO: PILOT PROJECT IN THE ANDARAÍ COMMUNITY
}

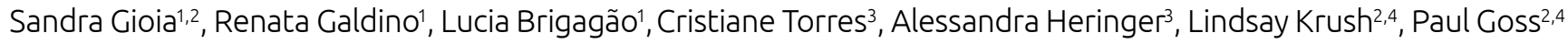

'State Secretariat of Health - Rio de Janeiro (RJ), Brazil.

${ }^{2}$ Global Cancer Institute - Boston (MA), USA.

${ }^{3}$ Andaraí Federal Hospital - Rio de Janeiro (RJ), Brazil.

${ }^{4}$ Massachusetts General Hospital - Boston (MA), USA.

This research project aims to study the increase of mammographic coverage rate with Patient Navigation Program (PNP) in a community of Rio de Janeiro. The general objective is to measure adherence to Ministry of Health's recommendations for breast cancer screening with mammography, with the help of a patient navigator. Other objectives include: 1) establish the viability of the PNP in this context; 2) identify the barriers to mammography screening and; 3) ensure that at least $70 \%$ of women recruited between 50 and 69 years of age and asymptomatic update the biennial mammographic examination and annual clinical examination, increasing the mammographic coverage from $14 \%$ to at least $70 \%$, which is the considered acceptable rate by the World Health Organization. From March to September 2018, women from the Andaraí Community were recruited in the Municipality of Rio de Janeiro. The follow-up was carried out by telephone, e-mail, and text messages. Of the 678 women (average age=58), 12\% refused to participate in the PNP for cultural reasons. All 599 women participants reported barriers to obtaining breast health care with the number of barriers reported ranging from 1 to 6 barriers, (average=3). The main barriers were system problems with health care programming (100\%), financial problems (64\%), communication concerns with the medical staff (58\%), fear (44\%), and social support (14\%). We obtained $100 \%$ satisfaction with the PNP and exceeded our goal with 88\% mammographic coverage rate. The PNP for breast cancer in the Andaraí Community has proven to be feasible in the context of local public health. The PNP promoted an increase in the mammographic coverage rate to $88 \%$, assisted in the transmission of quality information, reduced the fear of mammography, and facilitated access to breast health care. 